\title{
Mensaje político y eficacia electoral: microsegmentación en redes sociales en las elecciones seccionales de Quito
}

\author{
María José Calderón Larrea, Ismael Jaramillo Ampuero
}

Recepción: 9 de septiembre de 2019

Aceptación: 15 de noviembre

\begin{abstract}
El presente trabajo busca delimitar, en primer lugar, las características fundamentales que han girado en torno a los ejercicios de definición y uso de las plataformas digitales en campañas electorales. Para este fin, se analizará las particularidades del internet. En segundo lugar, se buscará comprender cómo este uso se ha ido implementando en el contexto ecuatoriano, con la finalidad de observar el trayecto de internet como medio de difusión masiva. Luego, se aproximará los mecanismos de estudio de mercado relacionados con las prácticas de microsegmentación, para, en último lugar, estudiar el caso de la campaña a la alcaldía de Jorge Yunda, el cual se ha configurado como un ejemplo de la aplicación, de manera eficaz, de la variabilidad del mensaje en el electorado, lo que ha sentado precedentes necesarios para definir futuras contiendas electorales.

\section{Abstract}

This article seeks to analyze the characteristics of digital platforms on electoral campaigns and the use of micro-segmentation of audience. To accomplish this first objective, this work will study the particularities of internet. Second, this study argues that this strategy has been successfully tried in the Ecuadorian context alongside variables of mandatory voting in small electoral districts. Moreover, it will analyze some market study mechanisms related with micro segmentation practices, which will lead to the analysis of the case study of Jorge Yunda's electoral run for mayor of Quito. His political campaign and further message have been an example of efficient micro segmentation and the adaptation of messages to social media applications. As a conclusion, this work will deal with the turnout of the increased impact of social media on politics and electoral results.
\end{abstract}




\section{Introducción}

L

a delimitación adecuada del electorado es un recurso vital para el éxito de los procesos electorales modernos. La diversificación de las tecnologías de la información logró que el intercambio de los acontecimientos se realice en tiempo real, lo cual hizo visible la necesidad de modificar los protocolos que desarrollaban la dinámica de la comunicación, produciendo así nuevos requisitos que vincularon al ejercicio de la política con variadas disciplinas (Hong, et al. 2016; Carañana, et al. 2018).

El retorno a la democracia en Ecuador (1979) y el posterior proceso estabilizador estuvo marcado por la pugna constante entre las distintas tendencias políticas (Izquierda, Derecha y Centro), la que desgastó en gran medida el ejercicio del poder y el manejo de "lo público". Los bajos índices de gobernabilidad y el estilo de gobierno determinaron la manera en la que el sistema se iba a establecer en los años posteriores, sentando así los precedentes para los altos y bajos que han caracterizado a los 40 años del retorno a la democracia en el Ecuador. La realidad política ecuatoriana, a lo largo de la historia, ha sido sumamente cambiante, en parte por los periodos de inestabilidad que ha caracterizado el desarrollo normal del sistema. Esto ha hecho que dadas las oportunidades brindadas por el sistema electoral, cada vez más actores políticos se vayan sumando a las contiendas electorales y que estas sufran modificaciones con la intención de fortalecer el ejercicio de la política (Pachano 1998: 22-43).

La variabilidad del contexto político electoral, sumada a la inclusión de nuevas tecnologías en la sociedad (principalmente las relacionadas con la comunicación), ha ocasionado que se modifique el modelo general de desarrollo de las contiendas políticas y electorales. Es por esto que los procesos generales de promoción de las tendencias electorales cada vez se dirijan más hacia lo digital, lo que ha impulsado un cambio obligatorio de discursos y de contenidos desde lo tradicional a lo digital; cuestión que se puede medir en la realidad ecuatoriana a partir del año 2012 -momento en el cual se puede hablar de una plena inclusión de internet en la política(Pachano 2007: 39-87). 
La transmutación del ejercicio político, junto a los beneficios de la revolución tecnológica, ha generado que las estrategias, la práctica, los presupuestos y los mensajes se direccionen hacia esta nueva vía que velozmente ha logrado revolucionar la realidad del ejercicio político ecuatoriano; además de generar rápidamente nuevos hitos que determinarán a futuro el ejercicio de la política. La digitalización de los espacios es el ejemplo máximo de los nuevos lugares de debate y de la inclusión de actores cada vez más diversos. En nuestro estudio recopilamos datos relacionados con la minería de redes sociales: Fechas de Inicio de posts, tipos de posts y número de seguidores.

Específicamente, recopilamos datos que hagan referencia a la utilización de Twitter como herramienta política. Durante el proceso de maduración de las redes sociales como herramienta política requirió el entendimiento de las ventajas y desventajas de estas como mecanismo concentrador de la opinión pública. Es por este motivo que la polarización de la opinión (mediante la presentación de ideologías extremas) se ha convertido en un recurso para aglutinar audiencia alrededor de un proceso de opinión.

El concepto de "cámara de eco" de internet, específicamente de las redes sociales, explica en buena medida el efecto concentrador de los mensajes concentradores, es decir: los mensajes polémicos tienden a ser más propensos a ser notados y reproducidos por las audiencias (Garmella, et al. 2018: 1-4). En ese sentido, las audiencias y los procesos de creación de nodos (con base al uso de "shares" en las publicaciones), han logrado que el alcance de un post polémico se incremente de manera exponencial.

Las nuevas revoluciones tecnológicas han modificado el concepto general de la sociedad global, constituyendo un "todo" que se encuentra más interconectado. Es entendible que este desarrollo provoque que el impacto de las Tic's produzca resultados que se correspondan con el desarrollo de distintos polos de crecimiento (económico, social, político), creando nuevos productos y servicios necesarios para su ajuste a las nuevas necesidades existentes (Castillo 2004: 4-7). 
El desarrollo de la economía alcanzado, más los nuevos niveles de accesibilidad e interconexión, no mide los presupuestos en los que se invierten los nuevos recursos obtenidos, es por esto que el alcance, beneficios y perjuicios del internet no fueron medidos de manera igualitaria, provocando que el desarrollo vinculado con lo digital no avance de la mano con los mecanismos adecuados de autocontrol y medición necesarios.

La utilización de las redes sociales, actualmente, no se puede considerar un fenómeno ajeno a los procesos clásicos de la comunicación. Esto ya que internet se ha incluido, de manera transversal, a todos los procesos básicos de la vida moderna. Es por esto que, junto al desarrollo de la red, se han desarrollado constantes estudios orientados hacia el análisis de su relevancia, lo que ha encontrado que existe una correlación entre clases sociales, niveles socioeconómicos y uso de Internet como herramienta. Yates y Lokley exponen la existencia del capital económico, la que influye en el flujo de utilización de redes sociales; tanto así que estas se han convertido en el nexo entre lo vivencial y lo digital (2018:1-4).

Esta investigación analiza dos variables fundamentales, el uso de redes sociales en una circunscripción particular de Quito y la adaptación del mensaje político. Nuestra hipótesis determina que el cambio en la comunicación política, a través de la microsegmentación de redes sociales en circunscripciones cerradas, y la obligatoriedad del voto son determinantes para obtener un triunfo limitado pero sostenido en una elección. Asimismo, este análisis definirá las categorías del mensaje político en Facebook y Twitter.

Literatura reciente demuestra la correlación entre la opinión pública polarizada, expresada a través de las redes sociales, y sus implicaciones en el proceso y la integridad de la toma de decisiones electorales y hasta de gobierno (Chen 2012).

Sugerimos que nuestros hallazgos tienen implicaciones más amplias sobre el uso de las redes sociales para la administración de una campaña electoral. Los resultados de nuestro estudio revelan la importancia de adaptar el mensaje político de los candidatos hacia las distintas plataformas del Internet social. El delicado balance del uso de redes sociales entre contenido 
promocional y el detonante de polarización política, lo que puede resultar nocivo en una campaña.

Para el entendimiento de internet, no podemos dejar a un lado sus principios fundamentales (Libre, Universal, Accesible, Anónimo, Inmediato). En ese sentido, es importante observar que el análisis costo beneficio, en la mayoría de los casos, tendrá un balance de suma positivo; sin embargo, se debe tener en cuenta que la capacidad de poner ante el público mayor cantidad de información no permitió que internet, por su desarrollo exponencial, pueda considerar protocolos de comportamiento y tratamiento de la información, creando así que la cantidad de datos a los que se puede acceder sean cada vez más grandes en información. Esto lo ha constituido como un derecho humano imprescindible para la evolución y crecimiento, lo cual se acompaña, de manera directa, con la utilización que se ha dado a este servicio por parte de la industria, ya que la cantidad de datos, y la inmediatez con la que el recambio se produce, ofrece fuentes de información a tiempo real que son medibles en el corto, mediano y el largo plazo (UN General Assembly Resolution: A/HRC/32/L.20 2016).

Los modelos de la comunicación en los que se aplicaba una dinámica direccionada, desde el centro a la periferia, generaron particularidades en las que los distintos elementos del diagrama de comunicación podían empezar a generar contenidos, lo cual produjo que los distintos miembros de estos nodos de interacción se empiecen a limitar mediante actividades y rasgos característicos. Esto produjo que los ejercicios de predictibilidad del comportamiento empiecen a tomar lugar, ganando así una importancia transversal en el ejercicio de la política. La orientación de mercado acompañó a la idea general de un mundo interconectado; esto ya que esta se conceptualizó a través de la creación de experiencias compartidas, lo que se acompañó con el principio de universalidad e inmediatez del Internet. La facilidad de almacenamiento de datos generó un nuevo mercado que puede ser explotado ante la compresión de las tecnologías, inaugurando una nueva forma de minería de rastros digitales. Todo esto significó un antes y un después en cuestiones de lanzamientos de campañas cada vez más específicas y personalizadas (Stieglitz y Dang-Xuan 2014). 
Existen poderes que temen al uso de la red, especialmente debido al desarrollo y evolución sin control que posee. Además, este se desenvuelve fuera de su área de influencia; sin embargo, no se debe olvidar que la red es también, en sí misma, una red de personas, lo cual la convierte en un sistema de asociación sumamente atractivo (Centurión 2006).

Es por este motivo que, en la actualidad, las dinámicas desarrolladas en la arena digital son, por demás, diversas. El conglomerado de usuarios se ha configurado como un elemento valioso para actores varios, lo cual ha generado nuevas prácticas orientadas a su manipulación y captación. La dinamización de los ejercicios generales de la comunicación en línea cada vez se configura como una disciplina en sí misma, migrando así de prácticas tradicionales como la mercadotecnia a un elemento nuevo, pero que adapta sus principios de ejecución más generales.

\section{Internet en la contienda política}

La Internet se configura como un conjunto de redes de carácter interconectado que posee sentido lógico, alcance global y dinámicas propias, su desarrollo se acompañó de la ruptura de los paradigmas tradicionales de la comunicación, cambiando el diseño clásico de su ejercicio a un proceso que vincula a las periferias con el centro. Es decir, una dinámica que establece procesos de comunicación desde audiencias pasivas hacia las audiencias activas.

Castells menciona que la Internet se configura como un elemento de información automatizado e interactivo por cuanto se configura a partir de mensajes direccionados en todos los sentidos posibles, esto produce que sean elaborados por cualquiera, se capten por cualquiera y no puedan ser organizados por nadie (Castells 1996: 77-82).

Las distintas dinámicas, ejemplificadas anteriormente, se han acompañado con ejemplos claros del carácter global del internet, el cual, en respuesta a sus beneficios, ha producido que se abra cada vez más al público general; este fenómeno se ha catalogado como la democratización de su uso y acceso. "La internet es el primer medio de comunicación de muchos 
para muchos. Esta fuerza y poder son no observante en temas relacionados a intereses y especulaciones" (Centurión 2006: 88-91).

La dinamización de la red y de los nuevos actores ha llevado a que plataformas tradicionales de la comunicación (prensa, radio, cine y televisión) migren sus audiencias hacia la red, lo que, en consonancia con la naturaleza sectaria de la humanidad, se tradujo en fenómenos de formación de grupos que se limitaron con base a un:

Constructo social, sumamente especifico. Las teorias sobre las revoluciones tecnológicas suponen un progreso hacia la desaparición de trabas y obstáculos. Una postura crítica, evidencia que el impacto tecnológico ha modificado las estructuras de relacionamiento comunicativo y que su resultado en el quehacer político aún no ha sido adecuadamente dimensionado (Calderón 2015: 10-13).

Según Puyosa (2015), es importante mencionar, y en atención a la formación de grupos símiles en la red, que existen distintas dinámicas que caracterizan a los movimientos colectivos en la red, las cuales son:

a. Arranque emocional de la movilización y elaboración de marcos de injusticia

Fenómeno que ante la visibilización de varias problemáticas logró que la asociación general sea un elemento frecuente en los debates de red.

b. Uso intensivo de la web y el teléfono móvil para la comunicación política autónoma

Ante la diversificación de la red, la inclusión de nuevas fuentes de acceso sea necesaria. Ante esto, el avance tecnológico produjo que los mecanismos de comunicación inmediata sean idóneos para esto, lo cual permitió que el intercambio se desarrolle en tiempo real.

c. Construcción de identidad colectiva a partir de marcos de valores y un lenguaje propio 
Los procesos asociativos han producido que la realidad de los fenómenos de movilización en la red produzca comunidades tradicionalmente poco factibles, esto ya que la cercanía, acceso y capacidad de intercambio de datos acercaron a perfiles similares.

\section{d. Debates sobre temas y valores enclaves deliberativos}

La capacidad de intercambio de ideas cree comunidades que trascienden a los constructos sociales tradicionales, al contrario, estas se forman con base a fundamentos teóricos que acercan más a los participantes.

\section{e. Acción política contra-públicos o contrahegemónico}

El rol abierto a debate de la red ha causado que los niveles de interacción permitan amalgamar fuertemente actores diversos. Esto ha hecho que la organización y la lucha en contra de temáticas específicas se haga más fuerte con el paso del tiempo y la vinculación de nuevos actores.

Estas ejemplifican de manera clara el rol inclusivo de la red, el cual se caracterizó por la difusión a largo plazo de sus ventajas. Esto fundamentó un escenario que se caracterizaría por la tendencia al crecimiento exponencial, provocando así que el direccionamiento de este servicio se oriente hacia la reducción de costos y masificación del acceso (Puyosa 2015: 197-209). Si bien es necesario entender que, al igual que en otros países, la utilización de internet ha sufrido un incremento exponencial, lo cual ha sentado precedentes para su uso normalizado, la lucha por la universalización de su uso ha provocado que este sea cada vez más atractivo para la difusión de contenidos de diversa índole, produciendo que nuevos actores, de relevancia y con capacidad integradora, se incluyan al debate público, enriqueciendo en gran medida el debate y la organización de audiencias. La importancia y facilidad de internet en favor de aglutinar audiencias fijas, las cuales poseen intereses y lenguajes comunes, no es un fenómeno nuevo; sin embargo, en el Ecuador no se puede hablar de un proceso de normalización de estas metodologías anterior a 2012.

Según lo expone Manuel Castells (1998), “el descubrimiento de la 186 
internet no fue sino un acontecimiento equiparable a la revolución industrial, esto ya que se configuró como un inductor de la base material de muchos de los polos de desarrollo de su tiempo". Interpreta a Internet como "el tejido de nuestras vidas (...) Internet es un medio para todo, que interactúa con el conjunto de la sociedad $\mathrm{y}$, de hecho, a pesar de ser tan reciente ha modificado las dinámicas clásicas del ejercicio de la comunicación y el diario vivir". Por lo cual, se comprende que este más que un sistema orientado para la comunicación se configura como medio de interacción y organización social (Castells 1998: 86).

Los nuevos ejercicios de comunicación, en los últimos 10 años, definieron reglas específicas en la conformación del diálogo en la red. Esto generó que, actualmente, todos los grupos de debate digital ganen importancia, determinando realidades que partidos políticos iban a tener que enfrentar en el corto, mediano y largo plazo. En este fin es necesario determinar las realidades de internet y como ha sido puesto en duda como medio de comunicación, debido a que, según la rama de estudio, internet puede ser considerado como un hipermedio o, en su defecto, un elemento que en su totalidad no llega a comprender todas las características esenciales de uno.

Como lo menciona Wolton, la dicotomía teórica existente en cuanto a la conceptualización específica de la internet se expresa en que, en primer lugar, en comparación con los medios de comunicación tradicionales (radio y televisión), internet no cumple con las características esenciales de uno (tecnológica, profesional y comercial), esto por cuanto no cuenta con la característica profesional que lo caracteriza, sino que se encuentra direccionado por grupos de personas de diversa índole (Wolton 2000: 18). Por otro lado, según Piscitelli, la red no solamente es un medio de comunicación; al contrario, esta se configura como un hipermedio por incluir a los medios de comunicación tradicionales mencionados con anterioridad, además de incluir nuevos; con base a esto, el autor expone que:

Internet, sin duda alguna, fue el primer medio masivo de la historia que permitió la horizontalización de las comunicaciones, se caracterizó por una armonía casi perfecta entre producción y recepción, alterando en forma indeleble la ecología de los medios (Piscitelli 1998: 207). 
Es con esto que podemos dejar sentadas las bases teóricas que delimitan, brevemente, a la internet y sus características; sin embargo, se debe tomar en cuenta la realidad acelerada de internet, la cual provocó cambios casi inmediatos en periodos de tiempo sumamente cortos. Por lo tanto, es complicado comprender de manera clara los efectos que esta ha tenido en la realidad social.

\section{Penetración de Internet en Ecuador}

El Internet en Ecuador ha tenido momentos marcados durante su proceso de asentamiento como herramienta básica de comunicación. Este proceso tuvo que irse adaptando a la realidad social, pasando de ser una herramienta de uso ocasional a ser una herramienta indispensable para el desarrollo de nuestras funciones diarias, lo cual modificó de manera exponencial la forma en la que se iban a desarrollar los procesos de nuestra vida diaria.

Esto nos permite entender que el ingreso de internet, como agente disruptivo, en el funcionamiento tradicional de los procesos sociales, económicos y políticos, puede ser considerado como un ejercicio de diálogo igualitario que generó nuevos niveles de inclusividad en la sociedad ecuatoriana (Calderón 2016: 65-77).

Ante esta afirmación saltan a la vista preguntas fundamentales, entre las que se encuentran, principalmente: ¿Cómo se ha manifestado la diversificación de internet en el contexto ecuatoriano? y si ¿Puede el proceso de interconexión jugar en contra de los usuarios digitales? La respuesta a estas preguntas sugiere que, una vez entendido el contexto ecuatoriano, se debe observar como los procesos de interconexión han jugado en contra de los distintos usuarios, esto aplicado a los nuevos procesos de targeting que se han ido normalizando en las prácticas, económicas, políticas y sociales.

El uso de tecnologías de la comunicación e Internet en la sociedad ecuatoriana no debe ser ajeno a la comprensión de la política como la capacidad de ser partícipe e incidir en los procesos de toma de decisiones en la esfera pública, y de lo político como el entorno en el cual la toma de 
decisiones tiene lugar. Con esto se busca decir que internet solamente se configuró como un mecanismo de carácter participativo que tuvo notable injerencia en la conformación del debate tras el poder, lo cual acercó los mandantes a sus representantes; no obstante, además de ser un mecanismo de presión política y social, no ha tenido mayor incidencia en el proceso real de toma de decisiones.

El caso ecuatoriano no dista de la realidad mundial, si bien el fenómeno del internet fue progresivo en las distintas regiones del globo. En un inicio, en el Ecuador, esta tecnología fue catalogada como un adelanto tecnológico que no tendría mayor trascendencia social, esto ya que solo podía ser utilizado por una élite específica (que poseía computadoras capaces de conectarse a la red); sin embargo, este número ha crecido hasta abarcar a casi la totalidad de la población mundial. Esto provocó que los Estados (partes fundamentales del Sistema Internacional de Estados) empiecen a mudar parte de su institucionalidad hacia lo digital.

Se ha hablado que el internet, como medio democrático, igualmente, ha desarrollado en base a la inclusión y el sistema de participación de actores tradicionalmente excluidos. Sin embargo, a nivel técnico y, en el marco del presente análisis, su conceptualización gira en torno a la aceptación de las particularidades individuales de cada grupo etario, es decir, Internet es lo que es gracias a su capacidad de adaptabilidad de las necesidades de los distintos usuarios que en el confluyen.

Esta aproximación permite que entendamos que a la vez que, en el contexto ecuatoriano, se incluyó a nuevos actores, estos se fueron agrupando según particularidades específicas acordes a su sistema de preferencias individuales, lo cual a su vez vuelve a sectorizarse con base a las características propias y determinantes del internet como medio de asociación masiva. Silverstone menciona que el estudio de los medios de distribución de contenidos se conforma como una manera de construcción propia de la sociedad contemporánea (2004: 32).

La utilización política de internet en campañas no es un fenómeno ajeno a la realidad ecuatoriana; no obstante, este, a lo largo de los distintos 
procesos, ha ido tomando un rol cada vez más importante en el campo de la configuración de la estrategia general de las campañas. Esto fundamentado en la facilidad de la red para poder perfilar similaridades en grupos particularmente extensos. Habermas menciona que:

La utilización del internet ha aumentado y fragmentado el contexto de la comunicación en si misma. Por lo tanto, este puede obtener efectos de carácter modificador de la realidad. Es por esto que, sus principios fundamentados en la informalidad, horizontalidad son características determinantes (...) el precio de esto es la obligatoriedad de la participación en tópicos de distinta índole; además del crecimiento en igualdad obtenido (Habermas 2006: 45).

Lo que nos permite inferir la verdadera determinación de este fenómeno tecnológico, mismo que ya ha trascendido a otros paradigmas de la vida humana. Los niveles de fragmentación del electorado ecuatoriano han representado un reto para cualquier figura con la pretensión de hacerse con un cargo de elección popular. Es por esto que la gran mayoría de los actores políticos, en los primeros momentos del uso político de la red, vertieron su atención hacia las redes sociales, empezando un mecanismo de flujo de recursos que se iba a acentuar con el pasar de los años y la profesionalización de los actores.

\section{Microsegmentación en Campañas Electorales}

Una vez tratados los dos puntos anteriores, es importante poner en contexto a la influencia del internet en los fenómenos relacionados con la comunicación política. La utilización de la red en política no significa más que "la práctica mediante la cual los distintos actores políticos buscan digitalmente el voto". Es por esto que las aproximaciones teóricas utilizadas anteriormente en favor de procurar definir al internet resultan sumamente útiles, por lo cual podemos decir que esta no se configura sino como un canal de intercambio de información, en tiempo real, de los distintos sucesos en la sociedad (Calderón 2015: 42-65). 
Los distintos ejercicios realizados por los actores vinculados al ámbito político, solamente, han adaptado prácticas tradicionales hacia lo digital, lo que nos permite hablar de un ejercicio de digitalización de métodos probados de la comunicación; sin embargo, este proceso de apropiación de lo digital ha venido acompañado con los distintos procesos de innovación fundamentados en las ventajas de la red; la utilización de estas ha diversificado el escenario del ejercicio de la política en tiempo real. Estas nuevas prácticas han producido "un ejercicio de cuestionamiento social hacia los medios tradicionales, lo cual ha desencadenado en su pérdida de legitimidad". Esto se puede traducir como un proceso de desgaste general en la emisión de contenidos, lo que se ha podido evidenciar en los procesos de movilización de contenidos desde lo tradicional a lo digital (Angulo, et al. 2008: 25-31).

Los actores vinculados a la política han tenido que maximizar su capacidad de adaptabilidad en los distintos procesos electorales (sumamente cambiantes con el paso de los ciclos electorales) que se han visto en la necesidad de maximizar los beneficios, economizando los distintos rubros invertidos, esto en vista de que la apertura electoral ha creado nuevos espacios para la difusión de propuesta y debate público atractivos para los distintos actores vinculados en la búsqueda del poder.

La popularización de las distintas redes sociales, de la mano con la "comunitarización" del debate en la red, ha logrado que nuevas estrategias comunicativas se encuentren a la orden del día, lo cual se ha configurado en un ejercicio de reconfiguración de preferencias, en el marco de un escenario en el cual la adaptación progresiva se convirtió en una necesidad para maximizar la utilización de medios digitales en la práctica efectiva de la comunicación política y la captación de públicos prioritarios.

Los líderes políticos no dialogan con el resto de los usuarios que recurren a Twitter y Facebook como mecanismo para la creación de estrategias de personalización a partir de narrativas consumibles, pero de rápida caducidad. En este marco, recurren a la construcción de enemigos ideológicos, más que personales (Angulo, et al. 2018: 13). 
Los procesos de amplificación discursiva de la mano de aplicación de los ejercicios de "ciberpolítica" (microsegmentación, geo politización y sobrecarga de mensajes) han llevado a que la búsqueda de la maximización se alcance en la menor cantidad de tiempo posible. Por lo tanto, la utilización de contenidos creativos, modificación del mensaje, personalización de intereses comunes, utilización de clips cortos con mensaje contundente y apropiación de temáticas populares, se han convertido en los nuevos recursos para la captación de votantes en las campañas políticas modernas (Cotarelo 2013: 292-293).

Las dinámicas de la red y, entre ellas, la capacidad de permanecer permanentemente conectado, migrando de plataforma de manera inmediata. Facebook, luego Twitter o Instagram permiten a los actores atacar permanentemente a segmentos del electorado mediante la aplicación repetitiva del mensaje (generación de narrativas personalizables), atendiendo la realidad de las audiencias de carácter nomádico. La construcción del mensaje en el marco de la microsegmentación del electorado no puede venir de un solo actor del ejercicio general de la comunicación de masas. Al contrario, esta debe ser un elemento generado a partir de las distintas interacciones de estos en torno a una problemática específica. Por lo tanto, el mensaje debe estar elaborado de acuerdo con las relaciones de los candidatos con el electorado, el lenguaje diseñado para la comunicación del mensaje y los distintos comunicados elaborados por los medios tradicionales.

Chen (2012) menciona que "la elaboración de meta-narrativas son el resultado de factores de carácter estructural y situacional (...) la elaboración del mensaje a partir de temas políticos claves termina cubriendo problemáticas generales de una campaña electoral (...)" (2012: 67-70). Con base a este postulado, es importante considerar que los nuevos ejercicios comunicativos buscan desvincularse del ejercicio tradicional de la difusión de mensajes comunes fundamentados en la adaptación del mensaje según la necesidad presentada.

Chen identifica tres pasos comunes que pueden ayudar a definir el uso de nuevos canales por las personalidades políticas, las cuales son:

- Modificación de las audiencias desde la masividad social, hacia prácticas más específicas y sectorizadas. 
- $\quad$ Adaptación del mensaje hacia audiencias locales.

- Utilización de las bondades naturales de cada plataforma.

Esto nos permite definir de buena manera que la comprensión del electorado como un agente sumamente volátil se ha convertido en una práctica necesaria ante la realidad generada por los procesos de micro asociación en la red (Chen 2012: 67-70).

Las distintas dinámicas generadas en los distintos procesos electorales en el mundo han dado cuenta de la importancia de la utilización de las nuevas plataformas. La variabilidad y capacidad de definición de la red hacia necesidades específicas ha producido que los roles en campaña política se vean, en gran medida, modificados.

\section{Las elecciones seccionales para alcalde en la ciudad de Quito en el año} 2019.

Esta sección buscará determinar los particulares que caracterizaron al ejercicio de delimitación de campaña en las elecciones seccionales 2019 y Consejo de Participación Ciudadana y Control Social. Como se ha mencionado con anterioridad, los ejercicios de definición objetiva de audiencias y los posteriores ejercicios de microsegmentación, se han convertido en alternativas para captar la mayor cantidad de electores en un proceso electoral, esto entendiendo la diversidad de este como un agente activo.

Los niveles de fragmentación registrados en las elecciones seccionales 2019 fueron un factor determinante para el resultado de este proceso electoral. La tabla número 1 nos permite observar la manera en la que se presentaron las votaciones, esto indicando los números obtenidos por los distintos candidatos. Del 100\% de electores, este proceso tuvo un nivel de participación del $81.79 \%$ (1'651.494) de la totalidad de electores habilitados (2’019.205). 


\section{Tabla 1:}

Votaciones obtenidas en las elecciones seccionales 2019

\begin{tabular}{|l|c|c|}
\hline \multicolumn{1}{|c|}{ CANDIDATOS } & VOTACIÓN & PORCENTAJE \\
\hline JORGE YUNDA & 296,096 & $21,39 \%$ \\
\hline LUISA MALDONADO & 255,007 & $18,42 \%$ \\
\hline PACO MONCAYO & 246,142 & $17,78 \%$ \\
\hline CESAR MONTUFAR & 234,422 & $16,93 \%$ \\
\hline OTROS & 619,821 & $25,48 \%$ \\
\hline
\end{tabular}

Al tomar en cuenta la evolución existente en los distintos procesos electorales en el Ecuador, es importante considerar la realidad que acompañó a las Elecciones Seccionales 2019 y Consejo de Participación Ciudadana y Control Social. Este proceso electoral se configuró como una contienda donde el entorno político -acarreado por las distintas configuraciones políticas- determinó la manera en la que el mensaje iba a ser estructurado; lo cual condicionó la forma en la que los electores se aproximaron hacia sus posibles autoridades y cómo, posteriormente, fueron captados por las distintas opciones electorales.

La adaptación de las campañas hacia un entorno político frágil produjo que el elector, en gran parte de los municipios, se vea influenciado por propuestas similares llegadas desde todos los bastiones políticos. Las distintas administraciones públicas que ocuparon el cabildo de la ciudad de Quito enfocaron gran parte de su gestión en acompañar o rechazar los lineamientos políticos imperantes en el país, lo que mermó las capacidades del municipio de generar obra y política pública eficaz durante su gestión. La situación política del Distrito Metropolitano de Quito fue un reflejo de la situación país. ${ }^{1}$

1.- Durante la campaña del 2019, medios de comunicación y actores de opinión instaron a la ciudadanía a encontrar las propuestas más relevantes, evidenciando la cantidad de mensajes y confusión en los mismos: https://www.planv.com.ec/historias/politica/quito-la-guia-electoral-elegir-al-nuevo-alcalde-metropolitano Inclusive desde instituciones públicas como la AME, se promueve la revisión de estos temas: https://amevirtual.gob.ec/cartilla-de-orientacion-para-organizaciones-politicas-y-ciudadania-elecciones-seccionales-ecuador-2019/ 
Considerando las particularidades de los procesos electorales ecuatorianos, la elección a la alcaldía del Distrito Metropolitano de Quito se ha configurado como un ejemplo claro de lo que la transmutación del mensaje puede hacer por la captación de un nicho de mercado. Para este fin, se analizará la campaña digital del actual alcalde de Quito, Dr. Jorge Yunda Machado, misma que se vio en la obligación de atacar diferentes segmentos del electorado; más aún cuando este se encontraba disperso por el gran número de candidatos a la Alcaldía y el contexto político ecuatoriano.

En las elecciones seccionales del 24 de marzo de 2019, la gran cantidad de opciones electorales provocaron que la participación política de la ciudadanía sea limitada y se disperse entre opciones poco capacitadas para ganar el proceso electoral. Entre los distintos candidatos que buscaban hacerse con la alcaldía del Distrito Metropolitano la división del electorado fue sumamente diversa, este factor que fue reflejado en las votaciones específicas obtenidas al final de la contienda electoral.

Tabla 2:

Construcción propia de los autores

\begin{tabular}{|l|r|c|}
\hline \multicolumn{1}{|c|}{ CIRCUNSCRIPCIÓN } & VOTOS & PORCENTAJE \\
\hline VOTACIÓN RURAL & 79363 & $20,7 \%$ \\
\hline VOTACIÓN URBANA NORTE & 130218 & $18,7 \%$ \\
\hline VOTACIÓN URBANA SUR & 86515 & $27,4 \%$ \\
\hline TOTAL & 296096 & $21,40 \%$ \\
\hline
\end{tabular}

La tabla número 2 muestra las votaciones del Alcalde Electo del Distrito Metropolitano de Quito, en estas se puede evidenciar que su votación, como candidato, fue sumamente diversa, asentándose en gran parte en la geografía de la ciudad. Sin embargo, esta votación refleja que el candidato no obtuvo buenos resultados en la circunscripción urbana del centro de Quito (En la que gana segundos y terceros lugares), en la cual no se hace con números positivos en cuanto a votación se refiere. 


\section{GRÁFICO 1}

Elección de alcalde en Quito-2019

Ganador por parroquia

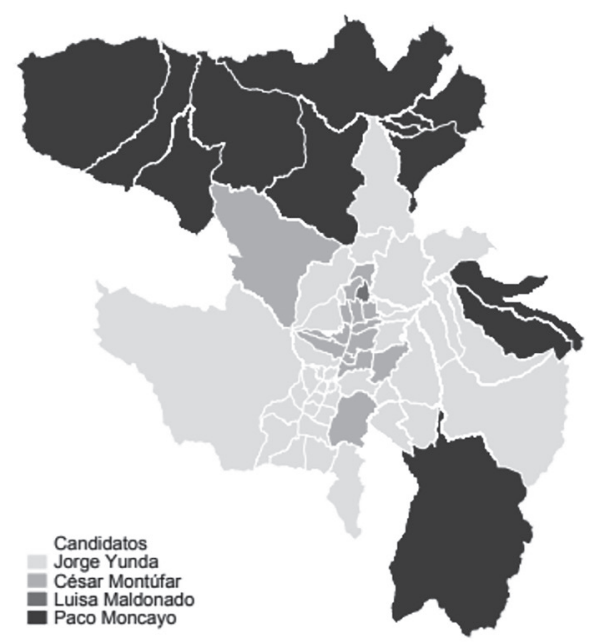

El gráfico número 1 nos permite observar la distribución de las votaciones aplicadas sobre el mapa de la ciudad de Quito, lo que nos permite comprender la dinámica general del proceso electoral y cómo esto determina el éxito de las distintas propuestas existentes.

\section{Gráfico 2:}

Penetración de internet en ecuador 


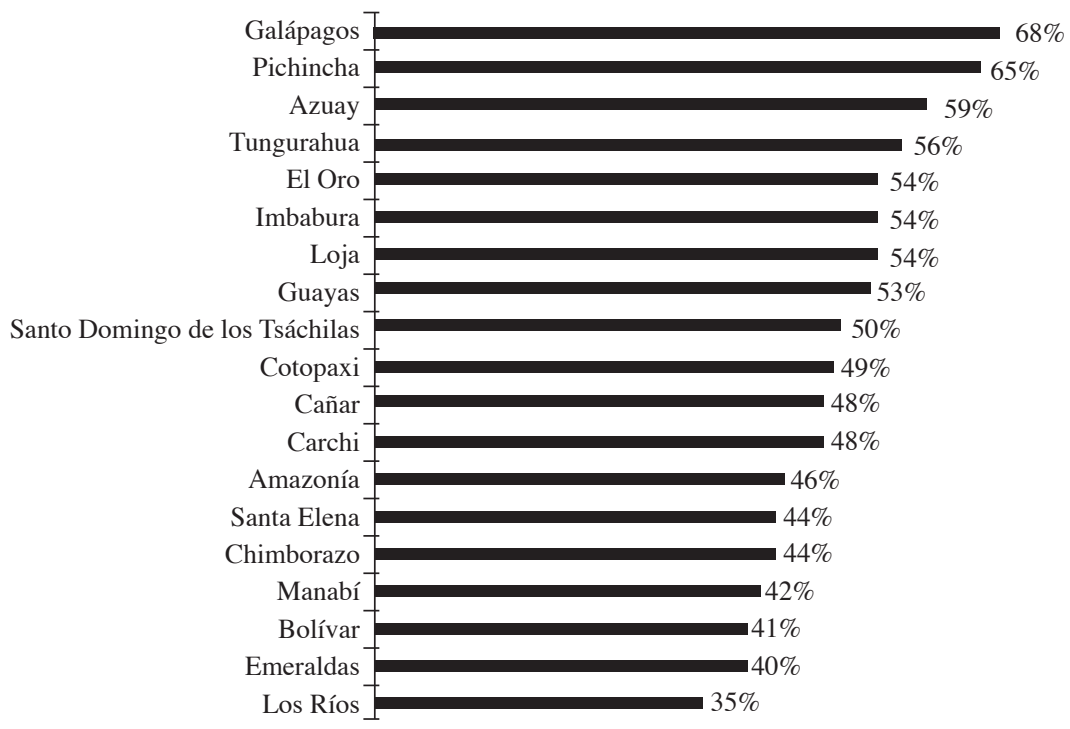

El gráfico número 2 ejemplifica los niveles de penetración de internet en el Ecuador, la homogeneización del acceso ha logrado que los sectores rurales cada vez se encuentren más incluidos en el debate de la red, lo cual puede explicar la gran aceptación de esta candidatura en la periferia de la ciudad; esto se puede observar de mejor manera en la tabla número 2 y el gráfico número 3.

\section{Tabla 3:}

Parroquias en las que ganó la candidatura de Jorge Yunda

\begin{tabular}{|l|l|}
\hline \multicolumn{1}{|c|}{ CIRCUNSCRIPCIÓN SUR } & \multicolumn{1}{c|}{ CIRCUNSCRIPCIÓN RURAL } \\
\hline CHILLOGALLO & ALANGASI \\
\hline GUAMANI & AMAGUAÑA \\
\hline
\end{tabular}


MARÍA José CALDERÓN LARREA, ISMAEL JARAMILlO AMPUERO

\begin{tabular}{|c|c|}
\hline LA ARGELIA & ATAHUALPA/HABASPAMBA \\
\hline LA ECUATORIANA & CALACALI \\
\hline LA FERROVIARIA & CALDERON \\
\hline LA MENA & CONOCOTO \\
\hline QUITUMBE & CUMBAYA \\
\hline SAN BARTOLO & CHAVEZPAMBA \\
\hline SOLANDA & CHECA \\
\hline TURUBAMBA & GUALEA \\
\hline CIRCUNSCRIPCIÓN NORTE & GUANGOPOLO \\
\hline COTOCOLLAO & GUAYLLABAMBA \\
\hline CARCELEN & LA MERCED \\
\hline COCHAPAMBA & LLANO CHICO \\
\hline COMITE DEL PUEBLO & LLOA \\
\hline EL CONDADO & NANEGAL \\
\hline IÑAQUITO & NANEGALITO \\
\hline JIPIJAPA & NAYON \\
\hline KENNEDY & NONO \\
\hline LA CONCEPCION & PACTO \\
\hline PONCEANO & PERUCHO \\
\hline RUMIPAMBA & PIFO \\
\hline SAN ISIDRO DEL INCA & PINTAG \\
\hline CHIMBACALLE & POMASQUI \\
\hline LA LIBERTAD & PUELLARO \\
\hline LA MAGDALENA & PUEMBO \\
\hline BELISARIO QUEVEDO & QUINCHE \\
\hline CENTRO HISTORICO & SAN ANTONIO \\
\hline CHILIBULO & SAN JOSE DE MINAS \\
\hline ITCHIMBIA & TABABELA \\
\hline MARISCAL SUCRE & TUMBACO \\
\hline PUENGASI & YARUQUI \\
\hline SAN JUAN & ZAMBIZA \\
\hline
\end{tabular}




\section{Gráfico 3:}

Porcentaje de votación de candidatos

Elección de alcalde en Quito-2019

Ganador por parroquia

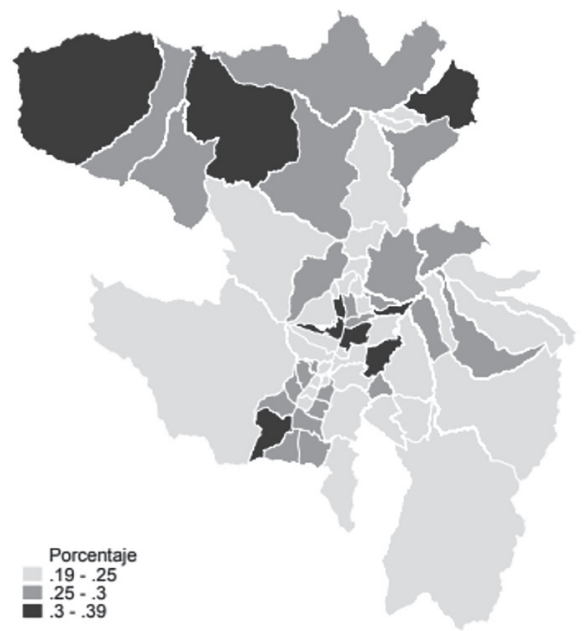

La captación del electorado según los datos presentados anteriormente busca delimitar cómo en el marco de los procesos de normalización del acceso y uso de internet, actores diversos se van incluyendo al debate ganando espacios de representación; sin embargo, los ejercicios de modificación del mensaje (de la mano con la microsegmentación de targets específicos) han permitido que los ejercicios generales de delimitación cada vez jueguen un rol más protagónico.

Nuestra metodología analiza la diferenciación entre tres redes sociales diferentes: Facebook, Twitter e Instagram. Cada una de estas tiene una diferenciación etaria no significativa en las circunscripciones analizadas; sin embargo, la división electoral de las parroquias urbanas obedece a diferenciaciones socioeconómicas bien definidas; siendo Facebook, la única que acapara el rango más grande de electores entre las circunscripciones de la ciudad de Quito. 
La tabla 4 nos permite sistematizar la mineraía de contenido, realizada a los mensajes de la campaña del actual alcalde Jorge Yunda. Se puede observar la modificación del mensaje en contextos específicos, para fin de este estudio. Este trabajo minó contenidos de las redes oficiales del candidato, considerando también aquellas que, mediante interacción, se convirtieron en elementos activos de campaña, estas son: @LoroHomero (página oficial en Twitter), @jorgeyundamachado (página oficial en Facebook), @ PanasJorgeYunda (página no oficial multiplataforma), @jorgeyundamachado (página oficial en Instagram).

\section{TABLA 4:}

Sistematización de mensajes vertidos en campaña

\begin{tabular}{|c|c|c|c|}
\hline FАСЕВООК & $\begin{array}{c}\text { TWITTER } \\
\text { JORGE YUNDA }\end{array}$ & $\begin{array}{c}\text { TWITTER PANAS JOR- } \\
\text { GE YUNDA }\end{array}$ & INSTAGRAM \\
\hline $\begin{array}{l}\text { Con más turismo } \\
\text { \#QuitoVuelveASon- } \\
\text { reír. Conoce nuestro } \\
\text { plan de gobierno } \\
\text { aquí... }\end{array}$ & $\begin{array}{l}\text { Esperaremos los } \\
\text { resultados en } \\
\text { casa, a que sea } \\
\text { lo que Dios y el } \\
\text { pueblo quiteño } \\
\text { decida. }\end{array}$ & $\begin{array}{l}\text { Los jóvenes tienen la } \\
\text { responsabilidad de elegir } \\
\text { a sus nuevas autoridades. } \\
\text { El Dr. Jorge Yunda dialogó } \\
\text { junto a \#GonzaloRosero en } \\
\text { @ democraciasec sobre sus } \\
\text { propuestas para la ciudad } \\
\text { \#QuitoGrandeOtraVez } \\
\text { \#JorgeYundaEs19 }\end{array}$ & $\begin{array}{l}\text { Gracias al periódico Metro } \\
\text { por permitirme transmitir } \\
\text { mis ideas y proyectos de } \\
\text { cara a lo que será mi admi- } \\
\text { nistración en la Alcaldía. } \\
\text { Entre otras cosas, yo quie- } \\
\text { ro un Quito sin miedo ¿y } \\
\text { tú? Conoce como lo logra- } \\
\text { relos en www.jorgeyunda. } \\
\text { com.ec \#QuitoGrandeO- } \\
\text { traVez \#YundaAlcalde19 } \\
\text { \#QuitoVuelveASonreír }\end{array}$ \\
\hline $\begin{array}{l}\text { Haré las obras que } \\
\text { Quito Necesita } \\
\text { para que todos sus } \\
\text { habitantes vivan } \\
\text { mejor. Desde marzo } \\
\text { cuando sea alcalde, } \\
\text { esa será mi mejor } \\
\text { publicidad \#Quito- } \\
\text { VuelveASonreír }\end{array}$ & $\begin{array}{l}\text { Siguen con las } \\
\text { noticias falsas, en } \\
\text { el entretenimiento } \\
\text { en Quito, debe } \\
\text { ser sin tortura sin } \\
\text { Maltrato animal, } \\
\text { la violencia } \\
\text { humana comienza } \\
\text { ahí. }\end{array}$ & $\begin{array}{l}\text { "El Metro debe ser decla- } \\
\text { rado como una obra de inte- } \\
\text { rés nacional para que pueda } \\
\text { seguir creciendo y sea } \\
\text { uyna verdadera solución } \\
\text { de transporte público para } \\
\text { Quito" \#QuitoGrandeO- } \\
\text { traVez \#JorgeYundaEs19 } \\
\text { \#YundaAlcalde19 }\end{array}$ & $\begin{array}{l}\text { Con un ambiente más } \\
\text { saludable \#QuitoGrandeO- } \\
\text { traVez \#YundaAlcalde19 } \\
\text { \#QuitoVuelveASonreír }\end{array}$ \\
\hline
\end{tabular}




\begin{tabular}{|c|c|c|c|}
\hline $\begin{array}{l}\text { Esta tarde el } \\
\text { legendario Coliseo } \\
\text { General Rumiñahui } \\
\text { se desbordó por el } \\
\text { entusiasmo y ale- } \\
\text { gría de las miles de } \\
\text { personas que lo co- } \\
\text { larón para respaldar } \\
\text { nuestra propuesta } \\
\text { de gobierno, basada } \\
\text { en planes realiza- } \\
\text { bles que mejorarán } \\
\text { sustancialmente a } \\
\text { nuestra ciudad y } \\
\text { la calidad de vida } \\
\text { de todos quienes la } \\
\text { habitamos \#Qui- } \\
\text { toVuelveASonreír } \\
\text { \#YundaAlcalde19 }\end{array}$ & $\begin{array}{l}\text { El verdadero } \\
\text { medio, aclara las } \\
\text { infamias que pu- } \\
\text { blican en contra } \\
\text { de una democra- } \\
\text { cia limpia }\end{array}$ & $\begin{array}{l}\text { Por invitación de los re- } \\
\text { presentantes de la @OEA } \\
\text { oficial, el Dr. Jorge Yunda } \\
\text { Machado @LoroHomero } \\
\text { dialoga con sus delega- } \\
\text { dos quienes garantizan la } \\
\text { transparencia del proceso } \\
\text { electoral de este domingo } \\
24 \text { de marzo, }\end{array}$ & $\begin{array}{l}\text { Tenemos un plan serio. } \\
\text { Nuestra campaña ha ido de } \\
\text { alegría, propuestas e ideas } \\
\text { \#QuitoGrandeOtraVez } \\
\text { \#YundaAlcalde19 \#Quito- } \\
\text { VuelveASonreír }\end{array}$ \\
\hline $\begin{array}{l}\text { Con más transpa- } \\
\text { rencia y un uso efi- } \\
\text { ciente de los fondos } \\
\text { públicos, \#Quito- } \\
\text { VuelveASonreír } \\
\text { \#YundaAlcalde19 }\end{array}$ & $\begin{array}{l}\text { Paseo obligado de } \\
\text { los sábados, buen } \\
\text { fin de semana a } \\
\text { todos }\end{array}$ & $\begin{array}{l}\text { "Hay } 600.000 \text { perros } \\
\text { deambulando por la ciudad. } \\
\text { Quito será una ciudad libre } \\
\text { de maltrato animal, porque } \\
\text { es ahí donde comienza toro } \\
\text { tipo de violencia" \#Quito- } \\
\text { GrandeOtraVez \#YundaAl- } \\
\text { calde19 \#QuitoVuelveA- } \\
\text { Sonreír }\end{array}$ & $\begin{array}{l}\text { El bienestar animal será } \\
\text { una de nuestras priorida- } \\
\text { des. \#QuitoVuelveASon- } \\
\text { reír }\end{array}$ \\
\hline $\begin{array}{l}\text { Combatiremos la } \\
\text { violencia de género } \\
\text { e intrafamiliar, a } \\
\text { través de planes } \\
\text { efectivos encabe- } \\
\text { zados por expertos } \\
\text { en la materia \#Qui- } \\
\text { toGrandeOtraVez } \\
\text { \#YundaAlcalde19 } \\
\text { \#QuitoVuelveA- } \\
\text { Sonreír }\end{array}$ & $\begin{array}{l}\text { Felicitaciones@ } \\
\text { Tame_EP }\end{array}$ & $\begin{array}{l}\text { Conoce de uentro plan de } \\
\text { trabajo en jorgeyunda.com. } \\
\text { ec \#Yundaes } 19 \text { \#YundaAl- } \\
\text { calde19 }\end{array}$ & $\begin{array}{l}\text { Desde la Alcaldía Pro- } \\
\text { moverá la inversión y el } \\
\text { desarrollo \#QuitoGrandeO- } \\
\text { traVez \#YundaAlcalde19 } \\
\text { \#QuitoVuelveASonre }\end{array}$ \\
\hline
\end{tabular}




\begin{tabular}{|c|c|c|c|}
\hline $\begin{array}{l}\text { Anoche me reuní } \\
\text { con los amigos de la } \\
\text { Zona Azul de Quito, } \\
\text { ante quienes expuse } \\
\text { nuestro plan de } \\
\text { gobierno y las solu- } \\
\text { ciones que daremos } \\
\text { a las problemáticas } \\
\text { que hoy los afecran } \\
\text { ¡Gracias por el } \\
\text { respaldo! \#Qui- } \\
\text { toGrandeOtraVez } \\
\text { \#YundaAlcalde19 } \\
\text { \#QuitoVuelveA- } \\
\text { Sonreír }\end{array}$ & $\begin{array}{l}\text { La vida y mi tra- } \\
\text { bajo me ha dado } \\
\text { la oportunidad de } \\
\text { tener más de } 400 \\
\text { compañeros en la } \\
\text { empresa, nunca } \\
\text { fueron emplea- } \\
\text { dos, respeto a sus } \\
\text { derechos }\end{array}$ & $\begin{array}{l}\text { "Invitamos a la ciudadanía } \\
\text { a revisar nuestro plan de } \\
\text { trabajo en jorgeyunda.com. } \\
\text { ec, tenemos un equipo de } \\
\text { trabajo técnico y profesio- } \\
\text { nal dispuesto a entregar } \\
\text { todo por esta \#QuitoQue- } \\
\text { rido@radiosucesosec } \\
\text { \#QuitoGrandeOtraVez } \\
\text { \#YundaAlcalde19 }\end{array}$ & $\begin{array}{l}\text { Esta tarde el legendario } \\
\text { Coliseo General Rumi- } \\
\text { ñahui se desbordó por el } \\
\text { entusiasmo y alegría de } \\
\text { los miles de personas que } \\
\text { lo colaron para respaldar } \\
\text { nuestra propuesta de } \\
\text { gobierno, basada en planes } \\
\text { realizables que mejorarán } \\
\text { sustancialmente a nuestra } \\
\text { ciudad y la calidad de vida } \\
\text { de todos quienes la habita- } \\
\text { mos \#QuitoVuelveASon- } \\
\text { reír \#YundaAlcalde19 }\end{array}$ \\
\hline $\begin{array}{l}\text { Con más actividad } \\
\text { física \#QuitoVuel- } \\
\text { veASonreír \#Qui- } \\
\text { toGrandeOtraVez } \\
\text { \#YundaAlcalde19 }\end{array}$ & & $\begin{array}{l}\text { Agradecemos a todo Quito } \\
\text { por escuchar las propues- } \\
\text { tas del Dr. Jorge Yunda } \\
\text { Machado @LoroHomero. } \\
\text { Este domingo 24 de marzo } \\
\text { reflexiona tu voto, elige } \\
\text { un cambio para lograr un } \\
\text { \#QuitoGrande OtraVez, } \\
\text { \#JorgeYundaAlcalde }\end{array}$ & $\begin{array}{l}\text { Es muy lindo recibir el } \\
\text { cariño de la gente mientras } \\
\text { hacemos algo que nos gus- } \\
\text { ta y nos llena el alma, en } \\
\text { mi caso, la música. Por eso } \\
\text { hay que apoyar a todas las } \\
\text { iniciativas culturales. Esta } \\
\text { noche de viernes tuve el } \\
\text { placer y el honor de volver } \\
\text { al escenario con Sahiro, en } \\
\text { los festejos por los } 50 \text { años } \\
\text { de la Asociación Ligas } \\
\text { Barriales de Pichincha } \\
\text { (ASOLIGAS) en Solanda, } \\
\text { institución a la que daré } \\
\text { todo mi apoyo en la ad- } \\
\text { ministración que tendré el } \\
\text { Honor de encabezar. COn } \\
\text { cultura y deporte, \#Quito- } \\
\text { VuelveASonreir }\end{array}$ \\
\hline $\begin{array}{l}\text { Cerramos la cam- } \\
\text { paña con alegría } \\
\text { con propuestas, } \\
\text { con proyectos, con } \\
\text { esperanza ¡Gracias- } \\
\text { Quito, gracias Sur } \\
\text { \#QuitoVuelveA- } \\
\text { Sonreír \#Quito- } \\
\text { GrandeOtraVez } \\
\text { \#YundaAlcalde19 }\end{array}$ & & $\begin{array}{l}\text { El Dr. Jorge Yunda atiende } \\
\text { a la prensa internacional, } \\
\text { @Telemundo, y expone } \\
\text { su plan de trabajo para los } \\
\text { hermanos migrantes, que } \\
\text { constituyen un importante } \\
\text { eje económico para la } \\
\text { capital ecuatoriana \#QUito- } \\
\text { GrandeOtraVez \#YundaAl- } \\
\text { calde19 }\end{array}$ & $\begin{array}{l}\text { Jorge Yunda: "Vamos a } \\
\text { convertir a Quito en la } \\
\text { ciudad más segura del } \\
\text { Ecuador. Ese es Nuestro } \\
\text { compromiso" \#QuitoVuel- } \\
\text { veASonreír, con más se- } \\
\text { guridad para todos. EN el } \\
2019 \text { ¡VOTA 19! Alcalde } \\
\text { Jorge Yunda, para volver a } \\
\text { sonreír. }\end{array}$ \\
\hline
\end{tabular}




\begin{tabular}{|l|l|l|l|}
\hline $\begin{array}{l}\text { Vamos por una } \\
\text { ciudad moderna, } \\
\text { con taxis adaptados } \\
\text { a las necesidades } \\
\text { actuales, con inno- } \\
\text { vación y excelencia } \\
\text { \#QuitoVuelveA- } \\
\text { Sonreír \#Quito- } \\
\begin{array}{l}\text { GrandeOtraVez } \\
\text { \#YundaAlcalde19 }\end{array}\end{array}$ & $\begin{array}{l}\text { "Que en este día de alga- } \\
\text { rabía democrática triunfen } \\
\text { las propuestas, las ideas, los } \\
\text { proyectos. Viva Quito, viva } \\
\text { Ecuador \#QuitoGrandeO- } \\
\text { traVez \#YundaAlcalde19 } \\
\text { \#JorgeYundaEs19 }\end{array}$ & $\begin{array}{l}\text { Como \#AlcaldeElecto les } \\
\text { extiendo mi mano, todos } \\
\text { los aportes son importan- } \\
\text { tes y serán considerados, } \\
\text { Juntos haremos un \#Quito- } \\
\text { GrandeOtraVez }\end{array}$ \\
\hline $\begin{array}{l}\text { Con bienestar ani- } \\
\text { mal, \#QuitoVuel- } \\
\text { veASonreír }\end{array}$ & $\begin{array}{l}\text { Perdemos alrededor de 3 } \\
\text { horas al día en el tráfico. } \\
\text { Solucionaremos de forma } \\
\text { definitiva este problema } \\
\text { que afecta la tranquilidad, } \\
\text { la productividad y el medio } \\
\text { ambiente de nuestra ciuda- } \\
\text { da" \#QuitoGrandeOtraVez } \\
\text { \#JorgeYundaEs19 }\end{array}$ & $\begin{array}{l}\text { Con un ambiente más } \\
\text { saludable para todos. } \\
\text { \#QuitoGrandeOtraVez } \\
\text { \#YundaAlcalde19 \#Quito- } \\
\text { VuelveASonreír }\end{array}$ \\
\hline
\end{tabular}

Entre los hallazgos más importantes del presente estudio podemos observar la manera en la que los ejercicios de modificación de contenidos toman lugar en la práctica. La campaña de Jorge Yunda aprovechó, en gran medida, el capital social que este tenía como figura pública, sin considerar que la campaña efectuada no tomaba en cuenta que el presente proceso electoral no era el primero para este candidato.

La campaña del alcalde Jorge Yunda centró sus lineamientos en tres ideas generales que giraban en torno a la trayectoria personal, profesional y ciudadana de este, las cuales son: Jorge en la música, Jorge en la Asamblea, Jorge el animalista y Jorge el ciudadano. Estos factores serían determinantes al momento de definir la parrilla de contenidos que la campaña trabajó durante el periodo de campaña electoral. Esta se definió según los usuarios de la plataforma, los que además de ser sujetos con distinto rango de edad, pensamiento político diverso y nivel sociocultural variado: se caracterizan por formar pequeños grupos de asociación dispersos por la red (fundamentados en intereses comunes) (Martínez 2014: 24-27).

El ejercicio de segmentación y microsegmentación obedeció a la 203 
comprensión de que el electorado que iba a participar en el proceso electoral era diverso y difícil de captar, factor que obligó a que la campaña realizada en el territorio se acompañe con una bitácora en tiempo real de las distintas actividades realizadas, esto con la finalidad de generar permanencia en el debate público para el mantenimiento adecuado del capital político.

Este ejercicio de análisis nos permite observar la manera en la que los ejercicios de complejización y simplificación de los mensajes, se han convertido en elementos claves para las victorias electorales. Estas prácticas han sentado un precedente claro en cuanto a la utilización de recursos de manera adecuada refiere, lo cual modificará a futuro hacia donde van a ser direccionados los capitales de campaña.

Las dinámicas de campaña generadas por Jorge Yunda en la elección 2019 se pueden ver ejemplificadas en las siguientes mediciones realizadas en los motores de definición de audiencia específica, estos indican:

\section{Gráfico 4:}

Perfiles con mayor crecimiento durante la campaña electoral 2019

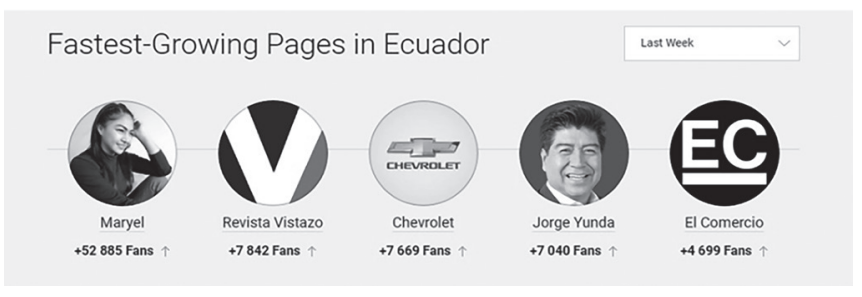

El gráfico número 4 nos permite observar los resultados obtenidos por la candidatura a la alcaldía del distrito metropolitano de Quito durante el tiempo de campaña, los cuales nos permite observar que el posicionamiento de "la marca Jorge Yunda Alcalde" se supo posicionar con muchas de las principales marcas a nivel nacional. 


\section{Gráfico 5:}

Perfiles con mayor crecimiento en ecuador durante la campaña 2019

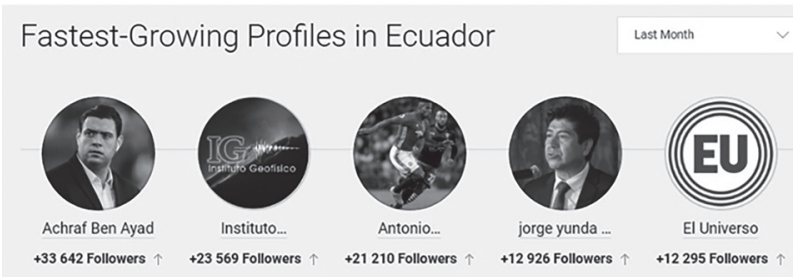

De igual manera, este ejercicio permitió que el crecimiento de Jorge Yunda se posicione con canales primarios de la comunicación (redes de canales de comunicación oficiales y medios de difusión de noticias) y perfiles de alto tráfico (Gráfico 5).

Las distintas plataformas tecnológicas permiten a los usuarios realizar ejercicios muy específicos de delimitación de las audiencias que estas quieren "atacar". Es por esto que el ejercicio preliminar de segmentación del público objetivo es un elemento sumamente atractivo para las distintas plataformas. Es por esto que los ejercicios de segmentación presentan las siguientes características.

\section{Grafico 6:}

Opciones de segmentación posibles en redes sociales

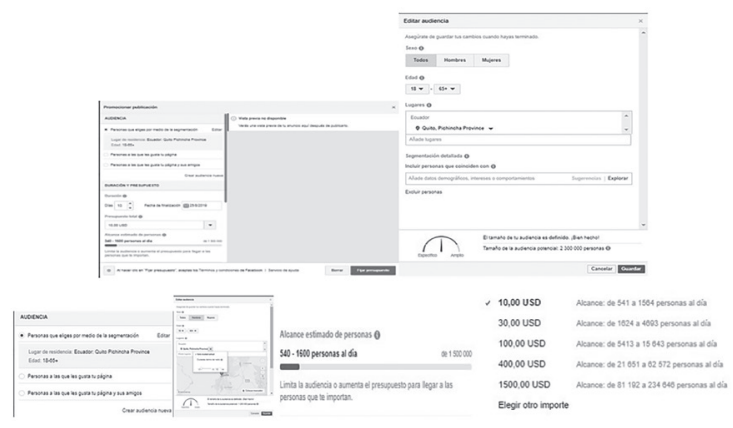


Las ofertas de segmentación de la mayoría de las plataformas ofrecen una delimitación adecuada del tipo de persona a la que una publicidad va direccionada. Es por esto que el amplio abanico de acciones posibles, permiten a atacar particularidades propias de un electorado ampliamente fragmentado. Es por esto que el ejercicio de definición de características específicas permite a las aplicaciones vinculadas al internet obtener un rol protagónico para este efecto.

La campaña a la alcaldía de Jorge Yunda se caracterizó por la importancia del mensaje de campaña, esto por cuanto a sabiendas de la dificultad para la captación del electorado. La tabla número tres, además de las definiciones estratégicas de mensaje (Jorge en la música, Jorge en la Asamblea, Jorge el animalista y Jorge el ciudadano), define cómo temáticas similares pueden ser tratadas según las particularidades de la plataforma, lo que se puede resumir de la siguiente manera:

- Facebook. - Red Social que durante la campaña se caracterizó por el rol político de las publicaciones, esta se fundamentó en la oferta de política pública y temáticas modernas. Este mensaje se delimitó en base a la gran popularidad de la red social dentro de los distintos segmentos de la sociedad.

- Instagram. - Es una Red Social que se utiliza conjuntamente con Facebook. Gracias a su simplicidad de segmentación, se configura como un recurso adecuado para quienes gustan de las ventajas del microblogging y la utilización de imágenes ilustrativas. En la campaña de Jorge Yunda sirvió como herramienta híbrida para quienes no fueron sectorizados por la campaña de Twitter.

- Twitter.- Es una red social que se caracteriza por la utilización del microblogging, la limitación en cuanto a la longitud de los tweets ha definido su mercado en un target específico; sin embargo, dado lo específico de su audiencia, durante la campaña de Jorge Yunda, ayudó en la difusión de mensajes con tendencias modernistas, factor que caracterizó la gran aceptación de esta candidatura en diferentes sectores de la ciudad. 
Se concluye, que los mensajes diferenciados, combinados con la multifacética personalidad del alcalde (quien contaba hasta ese momento con varios medios y un popular programa de radio); consiguió una votación sostenida por encima de las diferencias socio económicas de las circunscripciones electorales basadas en la ciudad de Quito y en base a la microsegmentación selectiva de mensajes y plataformas. Estas estrategias comunicacionales combinadas con los esquemas de comunicación política tradicional, construyó un voto constante (entre el 22-25\%) en una fragmentada votación, asegurándole su triunfo. 


\section{Bibliografía}

Angulo Moncayo, Natalia Alexandra; Arturo Virgilio Estrella Osorio y Marco López Paredes, (2018). "La política en Twitter. Un estudio comparativo de las estrategias discursivas de los candidatos finalistas a la Presidencia de Ecuador en 2017”. En: adComunica. Revista Científica de Estrategias, Tendencias e Innovación en Comunicación, ${ }^{\circ}{ }^{0}$. Castellón: Asociación para el Desarrollo de la Comunicación adComunica y Universitat Jaume I, 25-44. DOI: http://dx.doi.org/10.6035/2174-0992.2018.16.3

Carañana, Joan Pedro, Broud, Klaehn Daniel, Jeffery (2018). The propaganda model today. University of Westminster Press.

Calderón, María José (2015). Internet y política: deliberación, contienda y democracia en el ecuador 2007-2013: flacso - Sede Ecuador: Flacso Andes del Ecuador. 2015

Castells, M. (1998). La era de la información: economía, sociedad y cultura. México, D.F.: Siglo XXI Editores. 1999. v. 3261

(1996). La sociedad red. Madrid: Fondo de Cultura Económica.

Castillo, F. Dídimo (2004) "La Sociedad global y los desafíos para las ciencias sociales" Revista Venezolana de Ciencias Sociales, vol. 8, núm. 1, enero-junio, Universidad Nacional Experimental Rafael María Baralt Cabimas, Venezuela

Centurión, D. (2006). "Ventajas y riesgos del Internet como un canal de comunicación democrática". Revista Internacional de Investigación en Ciencias Sociales, ISSN-e 2226-4000, Vol. 2, No. 1,

Chen, P. (2012). "The new media and the campaign". In M. Simms \& J. Wanna (Eds.), Julia 2010: The caretaker election (pp. 65-84). Canberra: ANU Press. 
De Francis,G; K. Garmella; A. Gionis; M. Mathioudakis,; (2018), "Political Discourse on Social Media: Echo Chambers, Gatekeepers, and the Price of Bipartisanship".Academy of Finland Projects; Helsinki.

Martínez, M. L. (2014). Viralidad de los mensajes en Twitter en las Campañas Electorales [Virality of the messages on Twitter in the Electoral Campaigns]. III Congreso Internacional en Comunicación Política y Estrategias de Campaña, Asociación Latinoamericana de Investigadores en Campañas Electorales, Santiago de Compostela.

Cotarelo, R. (Ed.). (2013). Ciberpolítica. Las nuevas formas de acción y comunicación políticas. Valencia, España: Tirant Humanidades, 328 pp.

Habermas, Jürgen (2006). "Political Communication in Media Society: Does Democracy Still Enjoy an Epistemic Dimension? The Impact of Normative Theory on Empirical Research." En: Communication Theory $16411-426^{\text {a }}$ International Communication Association

Hong, Sounman, Hyoung, Kim Sun (2016) "Political polarization on twitter: Implications for the use of social media in digital governments". Government Information Quarterly Volume 33, Issue 4.

Pachano, Simón (2007) La trama de Penélope: Procesos políticos e instituciones en el Ecuador Quito: FLACSO Sede Ecuador : International IDEA : Institute for Multiparty Democracy.

(1998) La representación caótica: (análisis del sistema electoral ecuatoriano) Quito: FLACSO, Sede Ecuador.

Piscitelli, Alejandro. 1998. Post-televisión. Ecología de los medios en la era de Internet. Paidós. Contextos. Buenos Aires, Argentina.

Puyosa, I. (2015). “El campamento @PlazaResistencia en el ciclo de protestas en Venezuela 2014”. IDP. Revista de Internet, Derecho y Política, (21). 
Stieglitz,Stefan; Linh Dang-Xuan, (2014) "Emotions and Information Diffusion in Social Media-Sentiment of Microblogs and Sharing Behavior", in Journal of Management Information Systems, Volume 29, Issue 4.

Silverstone, Roger (2004). ¿Por qué estudiar los medios? Buenos Aires: Amorrortu.

UN General Assembly Resolution: 32/... The promotion, protection and enjoyment of human rights on the Internet: (Internet como un derecho Humano). A/HRC/32/L.20, 2016) General Assembly- United Nations. Tomado de: https://www.un.org/ga/search/view_doc.asp?symbol=A/ HRC/32/L.20 el 7 de noviembre del 2019.

Yates, S., \& Lockley, E. (2018). "Social Media and Social Class". American Behavioral Scientist, 62(9), 1291-1316. https://doi. org/10.1177/0002764218773821

Wolton, D; 2000; Internet. Petit manuel de survie Publicado por Sommerville House Books Ltd. ,Torornto, Ontario, Canadá (C) Flammario , París, 2000 